\title{
High-throughput quantitative detection of basal autophagy and autophagic flux using image cytometry
}

Gopika SenthilKumar', Justin H Skiba' \& Randall J Kimple ${ }^{\star, 1}$

\section{ABSTRACT}

Quantitative assessment of changes in macro-autophagy is often performed through manual quantification of the number of LC3B foci in immunofluorescence microscopy images. This method is highly laborious, subject to image-field selection and foci-counting bias, and is not sensitive for analyzing changes in basal autophagy. Alternative methods such as flow cytometry and transmission electron microscopy require highly specialized, expensive instruments and time-consuming sample preparation. Immunoblots are prone to exposure-related variations and noise that prevents accurate quantification. We report a highthroughput, inexpensive, reliable and objective method for studying basal level and flux changes in late-stage autophagy using image cytometry and acridine orange staining.

\section{METHODS SUMMARY}

A high-throughput, inexpensive, reliable and objective method for studying both basal autophagy and autophagic flux is reported. This approach uses acridine orange staining of late-stage autophagy and image cytometry to quantify autophagy.

\section{KEYWORDS}

acridine orange $\cdot$ autophagy $\cdot$ image cytometer

'Department of Human Oncology \& UW Carbone Cancer Center, University of Wisconsin School of Medicine \& Public Health, 750 Highland Ave, Madison, WI 53726, USA; *Author for correspondence: rkimple@ humonc.wisc.edu

BioTechniques 67: 70-73 (August 2019) 10.2144/btn-2019-0044
Macro-autophagy (referred to as autophagy hereafter) is a cellular recycling mechanism used for maintaining cytoplasmic homeostasis and surviving a variety of cellular stresses [1,2]. The most common inducer of autophagy is nutrient deprivation, although pharmacologic activation using the mTOR inhibitor, PP242, also induces autophagy (Figure 1) [3].

The first step in autophagy induction involves the formation of a doublemembraned autophagosome that engulfs cellular materials and subsequently fuses with the lysosome to form an autolysosome, where cellular materials are degraded [4]. Protein light-chain 3B-II (LC3B-II) is a key marker found on autophagosome membranes and its levels are studied using immunofluorescence (IF), immunoblot, flow cytometry and transmission electron microscopy (TEM) to understand changes in autophagy [2]. LC3B-II is degraded upon autolysosome formation, and thus inhibitors of autolysosome formation such as Bafilomycin A1 (Baf) are used to accumulate LC3-IIB for a standard amount of time to study autophagic flux $[2,5]$. However, the quantification of immunofluorescence is subject to imaging field-selection bias, counting bias, and observer variations even when using semi-automated quantification methods [6]. Immunoblot quantification is subject to incorrect results from minor loading errors and from oversaturation of house-keeping or target proteins in an attempt to distinguish differences in the protein of interest [7]. TEM requires specialized equipment and specific expertise for interpreting data and thus can be prone to misinterpretation [8]. Flow cytometry requires specialized equipment and time-consuming sample preparation, and is better suited for discerning the proportion of a sample that is undergoing autophagy. These methods are summarized and compared in Table 1.

Increases in autophagosomes can be caused by either autophagy induction or inhibition of downstream processing such as lysosomal fusion [2,9] (Figure 1). Therefore, it is important to analyze late-stage autophagy, such as changes in autolysosome levels, to directly study autophagic induction. Acridine orange (AO) is a fluorophore that accumulates in a protonated form inside acidic vesicular organelles (AVO) such as autolysosomes. At high concentrations, $\mathrm{AO}$ dimerizes, causing a metachromatic shift from green to red (Figure 1), which can be measured for studying late-stage autophagy. Thomé et al. can be referenced for detailed chemical and concentration changes [10]. AO can be assessed using fluorescence microscopy or flow cytometry, but these approaches suffer from the limitations described above (Table 1). We have used image cytometry as a quantitative, high-throughput, objective and inexpensive method (referred to as 'AO assay' from hereafter) that can be used to measure changes in basal autophagy and autophagic flux using $\mathrm{AO}$. The $\mathrm{AO}$ assay is a useful screening tool and a reliable, unbiased complement to the more qualitative and expensive approaches described above for studying autophagy.

UM-SCC47 cells were cultured in blackwall, clear-bottom 96-well plates (the outer wells were left empty as they were prone to noise/incorrect read-outs) and on eight-well IF slides. IF was used as a qualitative, established comparison for the AO assay. Cells were treated with DMSO, PP242 (500 nM or $2 \mu \mathrm{M})$ both with and without Baf (100 nM) for $24 \mathrm{~h}$. For IF 24-h post-treatment, slides were fixed and tagged with LC3B (D11) XP Rabbit mAb (Cell Signaling Technology \#3868), fluorescently conjugated (Alexa Fluor 488), stained with DAPI (Invitrogen Fluoromount-G with DAPI), and three random fields imaged using a $40 \times$ objective (Olympus BX41 inverted microscope with XM10 camera). The area of LC3B foci was quantified using FIJI and normalized to cell count [11]. A two-way ANOVA multivariance test (alpha .05) was performed using GraphPad Prism (GraphPad Software, 


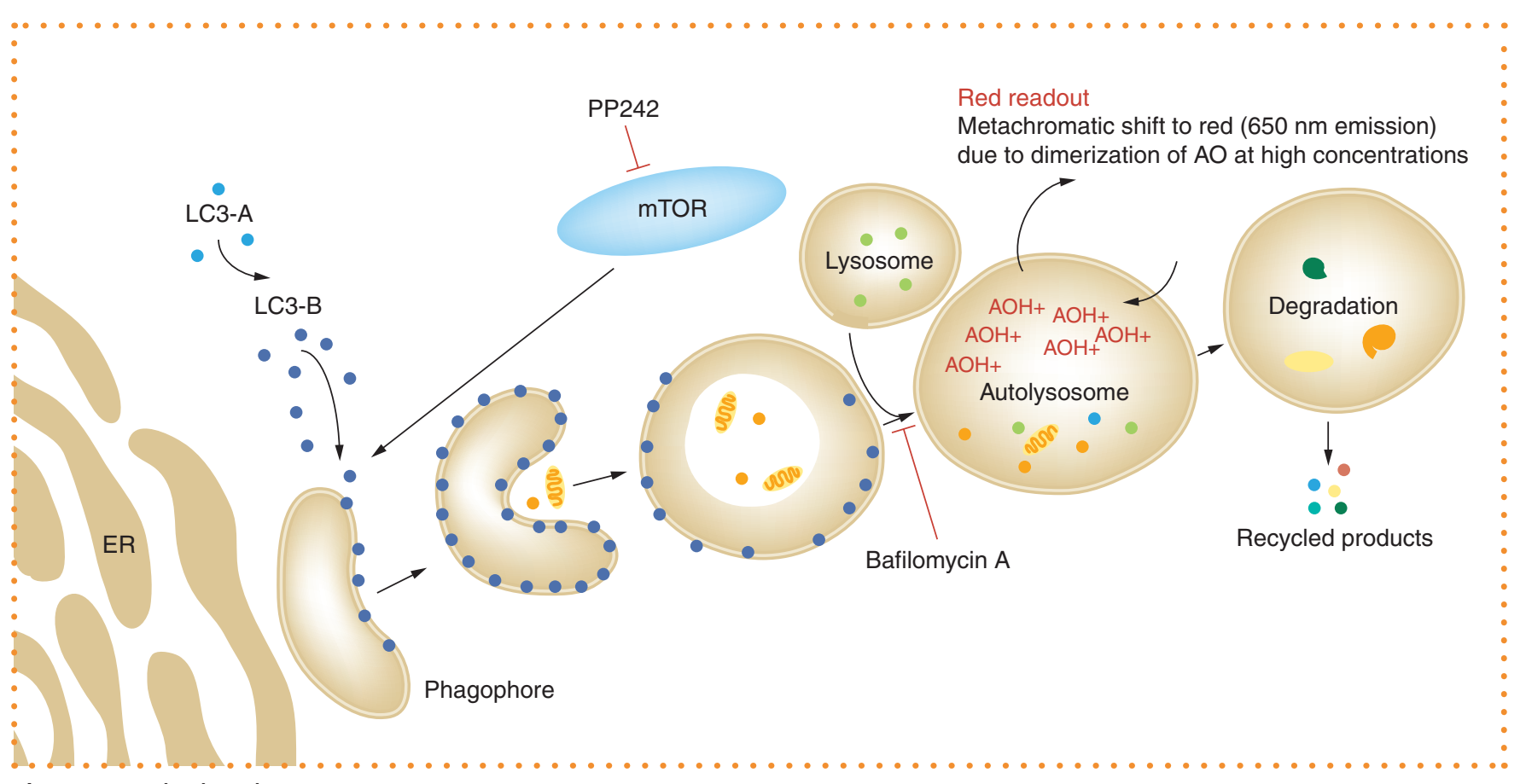

Figure 1. Autophagic pathway.

AO: Acridine orange; ER: Endoplasmic reticulum.

- Inc., CA, USA). Both basal autophagy (Baf-) and autophagic flux (Baf+ minus Baf-) was assessed (Figure 2A \& B). A different IF slide was washed with $1 \times$ phosphatebuffered saline at $\mathrm{pH} 7.4(1 \times \mathrm{PBS})$ and stained with $1 \mu \mathrm{g} / \mathrm{ml}$ AO solution diluted in $1 \times$ PBS. The slide was cover-slipped and immediately imaged as described above using a $20 \times$ objective (Figure $2 C \& D$ ).

For the image cytometer-based $A O$ assay 24 -h post-treatment, 96 -well plates were washed three times $(100 \mu \mathrm{l} /$ well $)$ with $1 \times$ PBS. $50 \mu \mathrm{l}$ of $1 \mu \mathrm{g} / \mathrm{ml}$ AO was added to each well and the plates were incubated at room temperature for $30 \mathrm{~min}$. Cells were then washed two times $(100 \mu \mathrm{l} /$ well) with $1 \times$ PBS. $50 \mu \mathrm{l}$ of $1 \times$ PBS was added per well, and then the plates were read on the SpectraMax i3 Multi-Mode Microplate Reader Platform with MiniMax 300 Imaging Cytometer (Molecular Devices, CA, USA) using the SoftMax Pro software (v6.4).
The monochromator and MiniMax settings were used on the software (Supplementary Figure 1). The monochromator setting was used to measure excitation/ emission wavelengths of 500/526 (green) to assess intensity of unprotonated, diffuse AO staining DNA (non-autophagic staining), and $460 / 650$ (red) to assess intensity of dimerized, protonated $\mathrm{AO}$ concentrated in AVOs (autophagic staining) [10]. The MiniMax setting was used to count the number of cells

\begin{tabular}{|c|c|c|c|c|c|c|c|}
\hline Analysis method & Result provided & $\begin{array}{l}\text { Sensitivity } \\
\text { for basal } \\
\text { autophagy }\end{array}$ & $\begin{array}{l}\text { Sensitivity for } \\
\text { autophagic } \\
\text { flux }\end{array}$ & Assay time & $\begin{array}{l}\text { Sample } \\
\text { prep. cost }\end{array}$ & $\begin{array}{l}\text { Instrument } \\
\text { cost }\end{array}$ & $\begin{array}{l}\text { Quantification } \\
\text { reliability }\end{array}$ \\
\hline $\begin{array}{l}\text { Immunofluorescence } \\
\text { microscopy }\end{array}$ & $\begin{array}{l}\text { Area or foci of } \\
\text { LC3B per cell }\end{array}$ & + & +++ & $\sim 8 \mathrm{~h}$ & ++ & +++ & ++ \\
\hline Western blot & $\begin{array}{l}\text { Specimen average } \\
\text { LC3B expression }\end{array}$ & + & +++ & $\sim 6 \mathrm{~h}$ & ++ & + & + \\
\hline Flow cytometry & $\begin{array}{l}\text { Average intensity } \\
\text { of LC3B or acridine } \\
\text { orange per cell }\end{array}$ & +++ & +++ & $\sim 4+h$ & ++ & +++ & ++++ \\
\hline $\begin{array}{l}\text { Transmission } \\
\text { electron microscopy }\end{array}$ & $\begin{array}{l}\text { Morphological } \\
\text { structures of au- } \\
\text { tophagosomes and } \\
\text { autolysosomes in } \\
\text { cells }\end{array}$ & ++++ & ++++ & $\sim 10+h$ & ++++ & ++++ & ++ \\
\hline Image cytometer & $\begin{array}{l}\text { Average intensity } \\
\text { of acridine orange } \\
\text { per cell }\end{array}$ & +++ & +++ & $\begin{array}{l}\sim 30- \\
45 \mathrm{~min}\end{array}$ & + & ++ & ++++ \\
\hline
\end{tabular}

Vol. 67 | No. 2 | $\odot 2019$ Randall J Kimple 


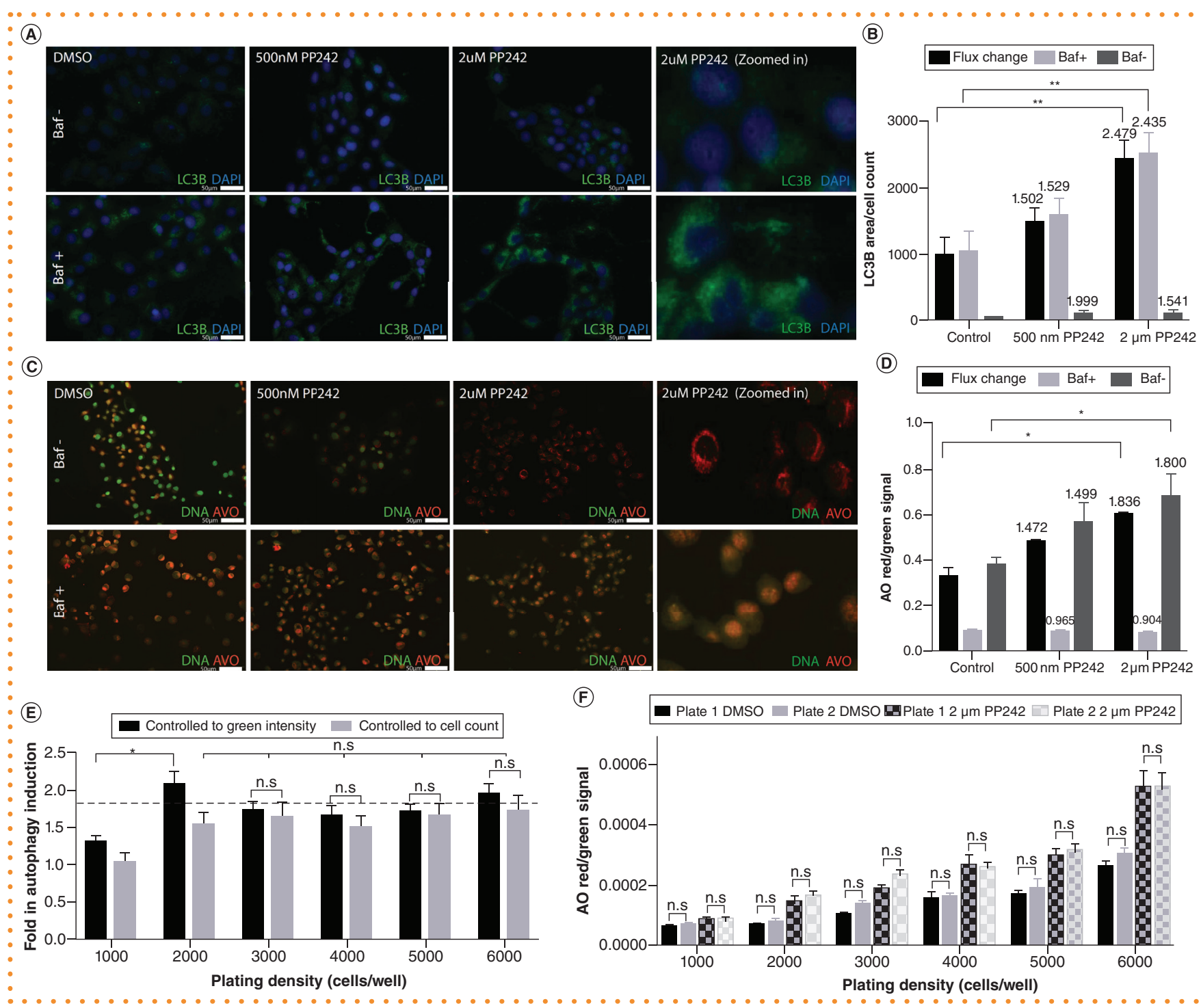

Figure 2. The analysis of acridine orange signal using the image cytometer provides an objective, high-throughput method for analyzing basal and flux changes in autophagy. (A) Representative 40x magnification immunofluorescence (IF) images tagged with LC3B antibody following treatments as indicated. (B) Average area of LC3B foci per cell $( \pm 95 \% \mathrm{Cl}$ ) measured using FIJI. The values above the bars indicate fold change. (C) Representative $40 \times$ images of AO-stained IF slides following treatments as indicated. (D) Average AO red/green signal ratio $( \pm 95 \% \mathrm{Cl})$ measured using image cytometer. The values above the bars indicate fold change. (E) Fold change results following $2 \mu \mathrm{M}$ PP242 treatment are consistent regardless of the normalization method used (cell count vs green intensity) at optimal plating densities. Dashed line shows the expected fold change from Figure 2D. (F) There are no significant differences in the average red/green signal ratio following $2 \mu \mathrm{M}$ PP242 treatment within the same treatment groups across multiple plates at varying cell densities.

${ }^{*} \mathrm{p} \leq 0.05 ; * \star \mathrm{p} \leq 0.01$.

AO: Acridine orange; $n . s$. : Nonsignificant.

per well as we have previously described [12]. The red intensity signal per well was divided by the green intensity or the cell count to assess the level of autophagy and to assess the efficacy of the two normalization methods: controlling to green intensity as recommended by Thome et al. versus controlling to cell count as previously done by Fowler et al. [10,12]. The statistical analysis performed on the IF data was performed on the $A O$ assay recorded values. Basal level autophagy and autophagic flux (Baf- minus Baf+) was graphed along with a $95 \%$ confidence interval.

As shown in Figure 2A \& B, imaging LC3B demonstrates a dose-dependent increase in autophagic flux but does not demonstrate a dose response in basal autophagy. However, the quantified results should be viewed with caution, as slight changes in the threshold used for quantification can drastically change the results (Supple- mentary Figure 2). We recommend using the IF images as a qualitative comparison. The graph in Figure 2B shows a significant increase in autophagic flux, but not basal level autophagy, following $2 \mu \mathrm{M}$ PP242 treatment. The higher fold change in basal autophagy after $500 \mathrm{nM}$ PP242 treatment compared with $2 \mu \mathrm{M}$ treatment, as well as the low LC3B foci areas, reflect the insensitivity of the assay to analyzing basal level changes. 
- Fluorescent images of AO-stained cells show a dose-dependent increase in basal autophagy (Figure 2C, top row), as illustrated by increased red intensity and decreased green intensity. In the Baf- group, the increase in red cytoplasmic intensity in conjunction with a decrease in green nuclear intensity with increasing doses of PP242 reflects a PP242 dose-dependent increase in basal autophagy [10]. In the Baf+ group, no significant differences are observed between groups, since Baf inhibits late-stage autophagy (i.e., accumulation of AVOs). Regardless, the Baf can be used for quantitatively studying autophagic flux (red/green ratio of Baf+ subtracted from Baf-; Figure 2D). The graph shows basal autophagy and autophagic flux measured by the image cytometer using AO (plating density: 5000 cells/well). An increase in both flux change and basal autophagy is seen following $2 \mu \mathrm{M}$ PP242 treatment, but not after 500 nM PP242 treatment. These data are consistent with the images in Figure $2 \mathrm{C}$ where a decrease in green but only a slight increase in red staining is seen after $500 \mathrm{nM}$ treatment. However, in the $2 \mu \mathrm{M}$ group, a clear shift from green to red is observed. The same trend is also seen in Figure $2 A$ \& B, with a clear increase in LC3B puncta following $2 \mu \mathrm{M}$ PP242 treatment, but not much change after $500 \mathrm{nM}$ treatment (Baf+ group). A correlation of autophagic flux between LC3B and AO shows an $R^{2}$ value of 0.9508 (Supplementary Figure 3), thereby confirming the sensitivity and accuracy of the AO assay. The efficacy of this assay was also confirmed using other cancer cell lines and other autophagy inducers/inhibitors (Data not shown).

To assess the effect of cell plating density and compare different normalization methods, we repeated the experiment using a range of plated cell densities (Figure 2E). At low densities, inconsistent results are seen (1000 and 2000 cells/well), while better consistency in this cell line is seen at plating densities between 3000 and 6000 cells/well. Similar results were seen independent of the normalization method used: green nuclear intensity versus cell count. Due to the significantly faster analysis using green intensity, this is our preferred approach. We also compared results across different plates and saw no consistent differences (Figure 2F).
These data demonstrate the value of image cytometry of $\mathrm{AO}$ to assess changes in basal autophagy and autophagic flux. The inexpensive nature of this assay, its ability to be used in high-throughput screens, and the objective nature of the data analysis make this assay a useful tool and complement to more qualitative approaches to studying autophagy.

\section{SUPPLEMENTARY DATA}

To view the supplementary data that accompany this paper please visit the journal website at: www.future-science. com/doi/suppl/10.2144/btn-2019-0044

\section{AUTHOR CONTRIBUTIONS}

GS and JHS performed the developed the project, performed the work, and drafted the manuscript. RJK oversaw all aspects of the work including project definition, methods and analysis, and manuscript writing.

\section{FINANCIAL \& COMPETING \\ INTERESTS DISCLOSURE}

Supported in part by grants from the American Cancer Society, the University of Wisconsin Carbone Cancer Center Support Grant (P30 CA014520), and the Wisconsin Head and Neck Cancer SPORE Grant (NIH P50 DE026787). The authors have no other relevant affiliations or financial involvement with any organization or entity with a financial interest in or financial conflict with the subject matter or materials discussed in the manuscript apart from those disclosed.

No writing assistance was utilized in the production of this manuscript.

\section{OPEN ACCESS}

This work is licensed under the AttributionNonCommercial-NoDerivatives 4.0 Unported License. To view a copy of this license, visit http://creativecommons.org/licenses/ by-nc-nd/4.0/

\section{REFERENCES}

1. Feng $Y, H e ~ D, Y a o Z$, Klionsky DJ. The machinery of macroautophagy. Cell Res. 24(1), 24-41 (2014).

2. Mizushima N, Yoshimori T, Levine B Methods in mammalian autophagy research. Cell 140(3), 313-326 (2010).

3. Zhou J, Tan SH, Nicolas V et al. Activation of lysosomal unction in the course of autophagy via mTORC1 作 Res. 23(4), 508-523 (2013).

4. Singh R, Cuervo AM. Autophagy in the cellular energetic balance. Cell Metab. 13(5), 495-504 (2011).

5. Tanida I, Minematsu-lkeguchi N, Ueno T, Kominami E. Lysosomal turnover, but not a cellular level, of endog84-91 (2005).
6. Jagoe $\mathrm{R}$, Steel $\mathrm{JH}$, Vucicevic $\mathrm{V}$ et al. Observer variation in quantification of immunocytochemistry by image
analysis. Histochem. J. 23(11-12), 541-547 (1991).

7. Bell G. Quantifying western blots: none more black. $B M C$ Biol. 14(1), 116 (2016).

8. Eskelinen EL. To be or not to be? Examples of incorrect identification of autophagic compartments in conventional transmission electron microscopy of mammalian cells. Autophagy 4(2), 257-260 (2008).

9. Yoshii SR, Mizushima N. Monitoring and measuring autophagy. Int. J. Mol. Sci. 18(9), 1865 (2017).

10. Thomé MP, Filippi-Chiela EC, Villodre ES et al. Ratiometric analysis of acridine orange staining in the study 4622-4632 (2016)

11. Schindelin J, Arganda-Carreras I, Frise E et al. Fiji: an open-source platform for biological-image analysis. Nat. Methods 9(7), 676-682 (2012).

12. Fowler TL, Bailey AM, Bednarz BP, Kimple RJ. High-throughput detection of DNA double-strand breaks using image cytometry. Biotechniques 58(1), 37-39 (2015). 